\title{
Derechos humanos en las políticas de paz y posconflicto en Colombia ${ }^{1}$
}

\author{
Human rights in the peace and post-conflict policy in Colombia
}

Sonia Patricia Cortés Zambrano ${ }^{2}$

Fecha de recepción: 15 de agosto de 2015

Fecha de aprobación: 7 de marzo de 2016

\section{RESUMEN}

Este artículo pretende reflexionar sobre los costos de la paz y el posconflicto en Colombia y la preocupación por la concreción o materialización real de los derechos humanos y la atención a grupos marginados. Dicha materialización se realiza mediante la adopción de políticas públicas con perspectiva de derechos y con amplia participación de la sociedad civil; de esta manera, los derechos humanos se convierten en el referente y fin último para las políticas públicas en materia de posconflicto. Y estas, a su vez, en el instrumento o medio idóneo para su realización. De este modo, el enfoque de los derechos de las poblaciones vulnerables debe ser una perspectiva para guiar la acción e intervención de las autoridades públicas, a través de los diferentes planes y programas de desarrollo económico y social.

Palabras clave: posconflicto, política pública y DD.HH., paz, víctimas, conflicto, política integral de paz.

1 El artículo es resultado de proyecto de investigación Institucional: "Derecho Humanos en las Políticas de Paz y Posconflicto en Colombia." en el marco del Grupo de Investigación de la Universidad Santo Tomás: "Doctor Angélico", Línea de Investigación: "Estado, Derecho y Constitución."

2 Abogada Universidad Santo Tomás, Especialista en Derecho Constitucional y Derecho Parlamentario de las Universidades Externado de Colombia y Autónoma de Madrid España, Magister en Derecho Público de las Universidades Santo Tomás y Universidad de Konstanz Alemania, Candidata a Doctora en Derecho de la Universidad Externado de Colombia, Docente Investigadora del Centro de Investigaciones jurídicas y políticas Francisco de Vitoria y Coordinadora del Semillero de Derecho Público: La Constitución y la Actividad del Estado en Colombia. E-mail: soniacortes@usantotomas.edu.co 


\begin{abstract}
Reflection falls on the costs of peace and post conflict in Colombia and concern for the realization or actual realization of human rights and attention to marginalized groups. This realization is made by the adoption of public policies with a rights perspective and with broad participation of civil society; thus, human rights become the reference point and ultimate goal for public policies on post-conflict and they turn on the instrument or suitable means for its realization. Thus, the focus of the rights of vulnerable populations must be a perspective to guide action and intervention by public authorities through the various plans and programs of economic and social development.
\end{abstract}

Keywords: Post-conflict public policy and human rights, peace, victims, conflict, comprehensive peace policy. 


\section{INTRODUCCIÓN}

El fortalecimiento y la solidificación de la paz para evitar una recaída en el conflicto es la preocupación institucional del Gobierno colombiano, intranquilidad que hace eco en la sociedad, en el sector privado, en las víctimas, en la clase política y académica del país. La pregunta de investigación de esta reflexión gira en torno a: ¿De qué manera la materialización de los DD.HH. en las políticas públicas son herramienta clave en el sostenimiento de la paz en Colombia? Y esta se resuelve en la tesis de reorganización en la distribución presupuestal, propia del ejercicio de planeación de los gobiernos regionales y el gobierno central, más allá del posconflicto, es decir como una dinámica de paz sostenible.

La construcción de la paz es un concepto inferior en extensión a "posconflicto" y abarca un mayor número de atributos, tales como: los procesos de desarme, desmovilización y reintegración de excombatientes, la reconciliación, la atención a población vulnerable, la construcción de memoria y verdad, la justicia transicional y la reparación, la prevención de la violencia y el crimen, la reforma de las Fuerzas Armadas y de Policía, la reconstrucción y el desarrollo económico, la estabilización política y la participación del sector privado, la sociedad civil y la comunidad internacional en todos ellos (Galtung, 1975; Boutros-Ghali, 1992; Chetail, 2009; Rettberg, 2003 y 2012).

No puede haber construcción de paz en medio del cruce del fuego entre actores. Pero sí podría hablarse de construcción de paz en zonas y espacios que no estén en medio de las hostilidades. Es decir, cuando existen ventanas de posconflicto (Ugarriza, 2013).

El posconflicto debe entenderse como el período de tiempo que sigue a la superación total o parcial de los conflictos armados. La rehabilitación posbélica es un término genérico que se refiere a la reconstrucción a largo plazo de la sociedad como consecuencia de conflictos violentos. Puede incluir cambios estructurales, de comportamiento y de actitud, por ejemplo al promover la reintegración o reinserción social, la reconciliación, la justicia restaurativa y la reconstrucción de la infraestructura física y de instituciones gubernamentales (Berghof Conflict Research, 2004). 
Se busca establecer un marco conceptual con relación a la dimensión del concepto, al entender este como parte integrante de un proceso más amplio de construcción de la paz. La construcción de la paz es un concepto que abarca todos los procesos, planteamientos, actuaciones, instrumentos y recursos necesarios para convertir los conflictos iniciales, y sus riesgos de devenir en violencia, en situaciones de paz estable, justa y duradera. Se concreta diferenciando tres momentos: el corto plazo (gestión de crisis y establecimiento de los acuerdos entre las partes), el medio plazo (rehabilitación posbélica) y el largo plazo (transformación del contexto, las actitudes, las conductas y las incompatibilidades que en su momento crearon, alimentaron y detonaron la violencia).

El manejo del período de posconflicto que ha seguido a periodos violentos se ha limitado a desarrollar una política de borrón y cuenta nueva, dejando de lado las necesidades de las víctimas y la reivindicación de derechos humanos. El diseño institucional cerró las posibilidades para un acceso democrático al poder y la falta de atención a las causas del conflicto generó nuevas violencias, por esto es necesario identificar las necesidades sociales reales con el propósito de hacer viables las políticas públicas de posconflicto.

La relación íntima entre derechos fundamentales, derechos humanos y políticas públicas es una preocupación constante a nivel regional y nacional. Se ha logrado la conquista de la positivización de los derechos humanos y fundamentales, pero falta su concreción real en directrices o políticas públicas que los hagan una realidad. Es precisamente la vulneración masiva y persistente de derechos humanos y derechos fundamentales la que genera movimientos sociales violentos, que atentan contra la dignidad humana, y la convivencia pacífica. Por eso, es allí en la verificación del incumplimiento o cumplimiento a medias de los deberes gubernamentales en materia de planeación, presupuesto y ejecución de políticas públicas en pro de las necesidades básicas de la población donde puede originarse el terror del conflicto y la imposibilidad de contrarrestar las acciones contrarias a la consecución de la paz.

Las nuevas funciones y nuevos roles de Estado determinan el derecho como herramienta para la realización de políticas públicas intervencionistas orientadas a la promoción de fines, valores e intereses sociales. (Calvo García, 2005. p. 10). 
El diseño de políticas públicas debe darse en el marco de un proceso de concertación entre el Estado y la sociedad civil, y debe dársele siempre el enfoque en derechos humanos y derechos fundamentales, teniendo en cuenta que estos se relacionan siempre entre sí. Debe considerarse, entonces, que los derechos humanos son el fundamento ético-moral y el objeto mismo de la acción del Estado y requisito para el bienestar de la sociedad. (Bernales, 2004).

Respecto a la realización de los fines del Estado, deben señalarse los principios que rigen la actividad del Estado y que deben ser prevalentes a la hora de planificar políticas públicas: el principio de la no discriminación, y de inclusión para la universalidad (juventud, género, infancia, etnia, etc), principio de dignidad humana, principio de democracia: una participación informada y protagónica, Responsabilidad compartida, prevalencia de la esfera pública, deliberación y resolución pacífica de los conflictos, y primacía de lo local en el ejercicio y realización de los derechos, dado que las acciones se dirigen a la realización de los derechos respecto a realidades (Tejada, 2004).

Podemos enmarcar algunas de las principales características de una política pública orientada al ejercicio efectivo de los derechos humanos y derechos fundamentales. Es decir, en procura de la materialización de la dignidad humana, con miras a evitar la recaída en relaciones de violencia y conflicto, originadas siempre en esa insatisfacción de la población que repara en sus condiciones de bienestar mínimo.

Integralidad: el diseño de políticas debe tener en cuenta derechos humanos y derechos fundamentales.

Intergubernamentalidad: las políticas públicas en derechos humanos deben permitir la articulación en los diferentes niveles de gobierno territorial -lo nacional, lo departamental y lo local-.

Coordinación: debido a lo anterior se necesita de la permanente concertación entre autoridades públicas de los distintos niveles de gobierno, en condiciones de respeto a la autonomía y bajo los principios de la concurrencia, coordinación y subsiedariedad de la acción pública. 
Intersectorialidad: las políticas públicas en derechos humanos comprenden acciones, planes y presupuestos de diferentes sectores y entidades públicas, las cuales deben actuar rompiendo el paradigma sectorial de la competencia por áreas.

Participación: las políticas deben ser elaboradas e implementadas con una amplia participación y consulta de las poblaciones beneficiarias o afectadas por la problemática (Garreton, 2004).

Para finalizar esta primera parte, las políticas deben ser, especialmente en materia de posconflicto, y en general siempre, políticas de promoción y cultura de derechos, políticas de prevención frente a vulneraciones, políticas de defensa y protección y políticas de concreción y materialización. Consisten en acciones tendientes a generar las condiciones que posibilitan el ejercicio pleno de los derechos humanos.

Hablamos sobre todo de políticas sociales en perspectiva de derechos humanos y son programas dirigidos a la búsqueda de la complementariedad y a la satisfacción integral de las personas: política educativa, política agraria, política en salud, política de empleo e ingresos, políticas redistributivas, política laboral, políticas de salarios, políticas de desarrollo humano, políticas de seguridad social, políticas de subsidios a grupos vulnerables, política alimentaria, etc.

Precisamente, para la concreción de políticas públicas en materia de derechos humanos con ocasión del posconflicto, el Gobierno nacional ha creado el Ministerio del Postconflicto, una entidad del nivel central que lidera el desarrollo de políticas públicas en materia de paz, en el marco de un Estado Social y Democrático de Derecho. El Ministerio se dedica a formular, adoptar, dirigir, coordinar y ejecutar la política pública en materia de paz y posconflicto en el marco de los acuerdos derivados de la mesa de conversaciones de La Habana, en Cuba, y de lo dispuesto por la Constitución y la ley.

El Ministerio se encarga de coordinar las relaciones entre la Rama Ejecutiva, la Rama Judicial, el Ministerio Público y los organismos de control, para el desarrollo y consolidación de la política pública en materia de paz y posconflicto a través de la 
Formulación, adopción, promoción y coordinación de las políticas y estrategias en: racionalización, reforma y defensa de la paz y los procesos propios del posconflicto.

El Ministerio tiene como objetivo dentro del marco de sus competencias formular, adoptar, dirigir, coordinar y ejecutar la política pública en materia de paz y posconflicto: planeación, ordenamiento jurídico, defensa y seguridad jurídica, acceso a la justicia formal y alternativa, lucha contra la criminalidad, mecanismos judiciales transicionales, prevención y control de la reincidencia, asuntos carcelarios y penitenciarios, promoción de la cultura de la legalidad, la concordia y el respeto a los derechos, la cual se desarrollará a través de la institucionalidad que comprende el Sector Administrativo.

La paz produce dividendos. La paz dinámica, la deseable para Colombia, es una ganancia de la nación al generarse riqueza en paz. El país gana al firmar el fin del conflicto, la ganancia es la riqueza que hoy deja de producir por el conflicto armado interno.

La paz debe ser prioridad de la agenda gubernamental, debe estar planeada y presupuestada, ya que se ha hecho un ejercicio para calcular cuánto va a costar la paz y el posconflicto. El análisis se divide en dos partes: conceptualizar la paz y el posconflicto y luego se revisar y socializar la planificación formulada por la comisión de paz, una comisión pluripartidista del Senado de la República, en contraste con la agenda del Gobierno aún desconocida en términos de lo que va a costar la paz en Colombia (Kalmanovitz, 2011).

No se pueden establecer los mismos recursos para lo mismo, esto no es invertir en paz. La paz requiere algo más: para hacer la paz deben medirse comunidades vulnerables, el analfabetismo, la desigualdad de género, la integración regional, la educación superior, la población carcelaria y su estado, entre otros. Es decir, debe realizarse un estudio diferencial del estado de los DD.HH., en los territorios vulnerables de Colombia.

El costo de la violencia en Colombia es de 50.000 millones de dólares al año, unos 100 billones de pesos. Y la inversión es de 5 billones de pesos, lo cual es realmente insuficiente en virtud a los recursos que deben ser destinados para generación de paz. 
Paz es el fin del conflicto, lo que cuesta transformar en paz el conflicto y generar pos conflicto es lo que cuesta sembrar la paz en las regiones y mantenerla después de la firma de los diálogos. Se requieren marcos institucionales, fortalecimiento institucional a todos los niveles y la creación de instituciones concebidas para administrar el posconflicto (Ley de transparencia, Ministerio del Posconflicto). Hay que construir esta institucionalidad en las regiones, en los departamentos y municipios, que hoy no cuentan con instituciones fortalecidas, ya que las instituciones del Estado son casi inexistentes en las poblaciones vulnerables y hay una ausencia de Estado (Cortés, 2012).

El esfuerzo por la convivencia pacífica es lo que debe facilitar el Estado, debe llegara las veredas, facilitar la conformación de organizaciones comunitarias con este propósito. Hoy el Estado se concentra en infraestructura y competitividad y este propósito llena la agenda pública gubernamental y no es el posconflicto y la paz los que ocupen el papel preponderante que debiera en el plan de desarrollo, el presupuesto nacional y la agenda del gobierno, lo cual no corresponde a la política reeleccionista.

Hay un compromiso gubernamental de priorizar la paz, y esta priorización se debe verificar en la planeación, en la destinación presupuestal para la paz, lo que no se aprecia.

La firma del acuerdo de paz, el compromiso de desarme, la desmovilización y la reinserción deben ser un hecho que debe verse presupuestado, y no lo está. Se necesita acompańamiento del Estado, y esto cuesta, requiere recursos. Para ejemplarizar, según datos (Álvarez \& Rettberg, 2007), dar de baja en combate a un guerrillero cuesta 6 millones de pesos, mantenerlo en la cárcel cuesta 12 millones, la resocialización cuesta 4,9. Es menos costoso resocializarlo, pero el proceso es largo, requiere tiempo y acompañamiento estatal. Hoy hay 4.738 casos reintegrados con éxitos (según agencia de la integración), $70 \%$ de los criminales procesados y encarcelados reincide y la reincidencia en los reinsertados es del $24 \%$. Por tanto, se trata de una decisión humana de reconstrucción del tejido humano y social.

Unos 630.000 millones de pesos requiere el posconflicto: garantizar inversión en jurisdicción agraria, en desarrollo agropecuario, formalización de propiedad rural y 
eficiencia tributaria, gobernabilidad local. Son 15 millones de colombianos víctimas del conflicto, situados en 325 municipios, quienes requieren reconstrucción del tejido social, sin contemplar a los desplazados que se encuentran en las grandes ciudades. Este presupuesto es para este grupo vulnerable de colombianos, lo que configura políticas públicas que deben planificarse, como el cumplimiento de los fines del Estado, con o sin política de paz, ya que constituye en sí misma un germen de paz. Es un deber del Estado para los municipios olvidados de Colombia.

La paz tiene cuatro componentes básicos que deben ser tenidos en cuenta al planificar políticas de paz, de posconflicto y de DD.HH.:

1. Concepción axiológica de la paz: es un valor fundamental, universal. La paz es el respeto a la vida, la adhesión profunda del ser humano a los principios de igualdad, libertad, justicia y solidaridad entre todos los seres humanos. Estos principios a su vez son los fundamentos del Estado Social de derecho y forman parte del juego de intereses que perfilan los fines esenciales del Estado. Cultura de paz según la ONU (1999) implica: Respeto a la vida, respeto pleno y promoción de los derechos humanos, arreglo pacífico de conflictos, protección del medioambiente, de generaciones presentes y futuras, respeto y promoción del derecho al desarrollo, respeto y fomento de la igualdad, respeto a la libertad, a la diversidad cultural, al diálogo y al entendimiento.

2. Dimensión institucional, pedagógica y comunicativa: es deber del Estado de educar para la paz. Fortalecer la cultura ciudadana, fomentar el conocimiento de los ciudadanos de sus deberes y derechos, así como de los mecanismos con que cuentan para la efectividad de los mismos. También la garantía del orden público, la redefinición del esquema de relaciones entre Estado, mercado y sociedad civil, fortalecimiento de las instituciones del liderazgo político, incluyendo la incorporación de la perspectiva de género como agenda política para la igualdad en razón a la eficiencia de derechos humanos y de cohesión social, revigorización y fortalecimiento institucional de los partidos políticos y refuncionalización de los mecanismos de representación.

3. Entendimiento de los ciudadanos de los acuerdos de paz, de sus implicaciones. 
4. Política pública de fortalecimiento institucional y organizaciones comunitarias, que implica que los 15 millones de colombianos marginados del Estado sean dueños de los procesos de desarrollo y construcción, y no beneficiarios.

No se evidencia asignación presupuestal o planeación presupuestal para el desarrollo de estos tres componentes. El presupuesto de 2015 no refleja este interés central del Gobierno, lo cual es contrario al discurso gubernamental. Debería estar planificado ya en una política pública. No basta con los subsidios, ni con la reparación administrativa de las víctimas, por lo que es necesaria la construcción de políticas públicas al respecto.

Qué es lo que no está previsto por el Gobierno y debiera estarlo en el marco para la paz:

a. Construcción del sistema jurídico para el posconflicto y adecuación de las instituciones jurídicas para enfrentar la situación social como una realidad constitucional.

b. Construcción de un fondo financiero exclusivo para la paz, con la construcción de un modelo de planeación, de seguimiento y de evaluación.

c. Organización territorial que garantice sinergias entre la paz local y la paz de las regiones, garantía de existencia y eficacia de la organización institucional para la paz en cabeza del Ministerio.

d. Sistema de inversiones públicas para la paz, lo cual garantiza que todos los Ministerios deban encaminar sus políticas a la paz y la garantía, protección y ejercicio de los derechos humanos.

Hay un déficit en Organización de Justicia transicional en las regiones, la jurisdicción agraria, jurisdicción de tierras, un desconocimiento en la situación real de la vivienda rural y las comunicaciones interveredales, y no se aprecia la existencia de una reforma tributaria para la paz. 
La institucionalidad local para los 325 municipios más afectados con la violencia tendría un costo de 90 billones de pesos a 10 años, y se consolidaría la paz a través de la lucha efectiva y eficiente del Estado contra la pobreza. Se requiere un plan de desarrollo para la paz y el posconflicto, el cual no existe hoy. Colombia deja de ganar 100 billones de pesos al año por cuenta de la guerra y el conflicto armado interno. La paz a 10 años puede costar 10 billones de pesos, por lo que se debe entonces luchar contra el verdadero enemigo de la paz que es la pobreza, la cual es responsabilidad de cualquier Gobierno vigente.

\section{Costos DE LA POLÍTICA INTEGRAL PARA LA PAZ}

Si se quiere en realidad construir una política integral de paz y posconflicto, el Estado debe tener claro que existen tres tipos de municipios con necesidades distintas que requieren intervención:

1. Las grandes ciudades que sentirán las secuelas de la desmovilización sin descuidar la seguridad ciudadana.

2. Al menos 600 municipios entre rurales, urbanos y ciudades intermedias, que igualmente son el eslabón a esa Colombia vulnerable del conflicto con los ciudades que gozan de instituciones de Estado.

3. Y 368 municipios más victimizados por la guerra: que padecen el rigor del conflicto, que conviven con las FARC, el ELN, las BACRIM, donde viven 15 millones de colombianos, la mayoría rurales. Ellos deben ser los prioritariamente atendidos por el Estado: los departamentos de Meta, Arauca, Caquetá, Cesar, Nariño, Chocó, Vaupés, Guaviare, Putumayo, Yopal, Guainía, Cauca, donde más del 50 \% ha sido azotado, población duramente afectada por el conflicto, con altísimos niveles de pobreza, desconexión del país en vías, con deficiente infraestructura social y desempleo.

Para que sus necesidades sean prioridad en la agenda gubernamental, nacional. 
Es indispensable para planificar la política pública lo siguiente:

Primero: priorizar las inversiones para la paz, la cifra de atención a municipios es de 368 municipios, para llegar a la Colombia rural y urbana.

Segundo: la Unidad de planeación ya no debe ser en el municipio, el Estado debe aprender a planear y a invertir según los centros poblados y las veredas rurales, para poder llegar y construir instituciones en esa parte de la población colombiana que hoy no tiene Estado. Se habla de 9.134 centros poblados y 87.342 veredas rurales en Colombia. La política de inversión para la paz debe estar dirigida prioritariamente a 4.254 centros urbanos y 40.039 veredas (Comisión para la paz del Senado de la República, 2014). Cambio de mentalidad política, que exige que el Estado y el Gobierno dirijan sus políticas a la totalidad del territorio nacional.

El Gobierno no está teniendo en cuenta a los 15 millones de colombianos a la hora de planificar. La paz no puede ser entendida como la desmovilización de 15.000 guerrilleros de las FARC, la paz es llegar a la mitad del territorio colombiano, a esos 15 millones de colombianos en sus necesidades básicas. De lo contrario, la estrategia para la paz está llamada a fracasar.

Se trata de cómo reemplazar las funciones del Gobierno o del Estado, que llegan a las poblaciones pobres, que son mayoría en Colombia, en forma de vacunas, cheques de familias en acción, donde el Estado actúa como una ONG, pero los que ejercen el poder, los que garantizan la seguridad, los que regulan, son los grupos ilegales en estos territorios. Esta es una redistribución perversa del poder en estos municipios.

Son entonces tres grandes ejes de inversión a saber:

1. Construcción de ciudadanía: garantizar construcción de institucionalidad, control social y político, reconstrucción de la memoria histórica, de sus instituciones, organizaciones que garanticen autorregulación. En estos lugares donde el Estado es ausente, los colombianos probablemente no han visto un fiscal, no hay un comisario de familia, porque los conflictos los resuelven los grupos al margen de la ley, el propósito es construir ciudadanía, formar 
ciudadanía, que confíe en la presencia efectiva del Estado. Reivindicar a esta población como sujetos de derechos y deberes, garantizarles el derecho a la voz y el voto, además de garantizarles el ser participantes activos en la toma de decisiones que les conciernen. Esta política pública costaría 20 millones de pesos por vereda y el Fondo de iniciativas ciudadana para la paz cuenta con 80.000 millones de pesos por año, para cubrir esas poblaciones. La primera estrategia que debe implementar el Gobierno de Colombia es que los colombianos sean sujetos de derechos y obligaciones, que puedan construir presupuesto participativo local. Hoy el Gobierno cuenta con 700 millones de pesos para la construcción de ciudadanía, esto debe revisarse.

2. Fortalecimiento del Estado: cambiar de la filosofía del Estado, la cual ha sido: hay que llevar el Gobierno central, el Estado, a las regiones. Se mandan funcionarios a capacitar en las regiones. Sin embargo, el Estado no se lleva, no se trasporta de Bogotá a las regiones. Si los programas se implementaran en las regiones con instituciones legítimas que los defiendan, con funcionarios de la región conocedores de sus zonas, no habría esta ausencia de Estado. Los programas y las instituciones deben construirse en las regiones, con gente de las regiones. La lógica de intervención del Estado está fracasada debe cambiar. El Estado en la región es la alcaldía, por lo que se debe fortalecer el gobierno municipal, se debe invertir en el fortalecimiento institucional de los municipios. El Gobierno debe financiar a los municipios al $100 \%$ sin esperar contrapartida, por lo menos en:

a) Formación y actualización de su catastro urbano y rural en 368 municipios. Se calcula que 13.000 millones de pesos cuestan actualizar el catastro de estos municipios en 4 ańos.

b) Formalización de la propiedad hasta que se llegue al registro del título. Se calculan que existen 291.000 predios sin registrar, una cifra que se corroborará con el censo nacional agropecuario, que permitirá conocer el número total y real de predios. El costo aproximado será de 109.000 millones de pesos para legalizar las tierras. Los municipios deben recaudar recursos propios, pero si no tienen catastro, ni titulación de los predios, 
¿cómo se recaudan los impuestos prediales? Esto constituye perdida presupuestal para el municipio.

c) Creación del equipo de personal capacitado que colabore con personal cualificado que quiera trabajar en estos municipios, pero con la tarea esencial de capacitar y formar el personal con el talento humano propio de la región, y fortalecer la función tributaria. Que tengan la capacidad de gestionar sus propios recursos. Esto cuesta 5.800 millones de pesos. La autonomía consiste en que cada municipio tenga como vivir del recaudo de sus propios recursos, del desarrollo de su propia economía.

La suma a cuatro años para estos 368 municipios y 40.000 veredas cuesta 691.000 millones de pesos: solo una entidad del Estado -la DNP- tiene este presupuesto, y este proyecto de paz lo merece. Construir institucionalidad local le cuesta al Estado el presupuesto de una entidad del Estado.

3. La construcción de Equidad e inclusión: si no se construye ciudadanía y se fortalecen las alcaldías como gobierno local, además de empoderar a la ciudadanía, el dinero se pierde en burocracias y corrupción. Las inversiones deben centrarse en salud, en técnica agropecuaria, para poder planificar y ejecutar estas políticas en forma eficiente. Debe pensarse que por cada 50 familias se tenga un técnico agropecuario y un promotor de salud, remitidos a los centros de salud que hay en las cabeceras municipales. Se necesitan entonces 13.000 técnicos, 13.000 mil promotores de salud y 2.500 médicos.

En los próximos 10 años construir ciudadanía vale 900.000 millones de pesos, que implica la construcción de un fondo para la paz de iniciativa ciudadana. Construir instituciones locales costará 12.7 billones de pesos y lo que más cuesta es la equidad, agua, vivienda, salud, electrificación, técnica agropecuaria y educación: 79 billones de pesos en los próximos 10 años (Comisión de paz Congreso de la República, 2014).

En total, lo que se llama políticas públicas para la paz, que no es el documento de acuerdo de La Habana, implica la satisfacción de necesidades básicas de 15 millones 
de colombianos para concretar sus derechos fundamentales en virtud a los principios que rigen los fines del Estado: justicia, libertad, igualdad, solidaridad, bien común, dignidad humana (Roth, 2006), cuyo costo es de 93 billones de pesos a 10 años. Se requiere, como en todos los países, unas reglas fiscales para la paz. Los impuestos tendrán que impulsar y asegurar la financiación de la paz, y se debe fortalecer la tributación nacional y regional. La paz requiere una regla fiscal para la paz. El gobierno debe asegurar la financiación y la capacidad tributaria nacional y local para garantizar los recursos.

\section{Conclusiones}

En conclusión, se requiere una política visible del gobierno nacional, que responda a estas necesidades. Debe realizarse un esfuerzo en cumplir con el principio de redistribución equitativa de la riqueza, revisarse el plan nacional de participaciones y el plan de desarrollo; es una tarea para todos los entes de decisión política del Estado. Se cuenta con inversión extranjera que debe acompañarse de estrategias de control y vigilancia para garantizar su transparencia, se deben reforzar políticas de seguridad y garantizar la continuidad de las políticas de paz.

Las políticas de paz del Gobierno deben enmarcarse en el enfoque de los derechos humanos, en pro de su materialización y la garantía de condiciones de vida digna. Esto explica su cercanía con los procesos de desarrollo humano y socioeconómico. Las políticas públicas son, por tanto, instrumentos para la concreción de derechos humanos y la realización del derecho útil. Este enfoque permite ir más allá de identificar los derechos humanos con el conflicto armado interno y las violaciones, a una mirada más amplia que los concibe desde la aspiración humana con tener cubiertas sus necesidades básicas en condiciones propias de la dignidad humana.

La nueva visión de los derechos humanos y la construcción de políticas públicas de paz superan la visión negativa de los mismos (violaciones, vulneraciones y privaciones), para plantear una visión positiva: su protección, concreción y realización efectiva, en donde la intervención pública es vital para el desarrollo de los mismos, programas de acción pública que tienen como fin último el logro de los derechos humanos. 


\section{REFERENCIAS}

Álvarez \& Rettberg (2007). Cuantificando los efectos económicos del conflicto: una exploración de los costos y los estudios sobre los costos del conflicto armado colombiano. Colombia Internacional. Enero-Junio. pp. 14-37. Recuperado de: http://colombiainternacional.uniandes. edu.co/view.php/305/view.php

Bernales, E. (2004). Las políticas públicas desde la perspectiva de los derechos humanos. Lima: Comisión andina de juristas. pp. 93-109

Boutros, B. (1992). An agenda for peace. Nueva York: United Nations.

Calvo, M. (2005). Transformaciones del Estado y del Derecho. Bogotá: Universidad Externado de Colombia.

Cortés, S. (2012). Poder discrecional de la Corte Constitucional en el estado de cosas inconstitucional. Bogotá: USTA.

Chetail, V. (2009). Post-conflict peacebuilding: A lexicon. Nueva York: Oxford University Press. Traducción Universidad del Rosario. Colombia

Garreton R. (2004), "La perspectiva de los derechos humanos en el diseño y ejecución de las políticas públicas", en: Garretón, R. et. al. El enfoque de los derechos humanos en las políticas públicas, Lima, Comisión Andina de Juristas, pp. 27-48.

Galtung, J. (1975). Three approaches to peace: Peacekeeping, peacemaking and peacebuilding. Peace, war and defence. Essays in peace research, 2, pp. 282-304. Copenhague: Christian Heljers.

Kalmanovitz, S. (2011), El impacto económico del conflicto interno colombiano y un escenario de paz. Recuperado de: http://pnud.org.co/img_upload/3635346361636163616361 6361636163/El_impacto_económico_del_conflicto_interno_colombiano_y_un_escenario_de_paz_Salomón_Kalmanovitz.pdf 
Rettberg, A.. (2003). Diseñar el futuro: una revisión de los dilemas de la construcción de paz para el post conflicto. Revista de Estudios Sociales, 15, pp. 15-28.

Rettberg, A. (2012). Construcción de paz en Colombia: contexto y balance. En Rettberg. A. (comp.) Construcción de paz en Colombia. Bogotá: Ediciones Uniandes.

Roth, A. (2006). Discurso sin compromiso. La politica pública de derechos humanos en Colombia. Bogotá: Ed. Aurora.

Secretaría Senado de la República (2014). Comisión para la Paz. Debate parlamentario. Recuperado de: http:/www.senado.gov.co/historia/item/20835-plenaria-de-senado-analizo-financiacion-del-posconflicto (accedido en marzo 2015)

Tejada, D. (2004). Políticas públicas y derechos en salud. Recuperado de: www.minsa.gov.co/ documentos/informacioncursosextension.pdf. [Accedido en enero 2015].

Ugarriza, J.E. (2013). La dimensión política del postconflicto. Avances conceptuales y empíricos. Colombia Internacional, 77. pp. 141-176. 